\title{
Design of conversational agents for CSCL: comparing two types of agent intervention strategies in a university classroom
}

\author{
Konstantinos Michos, Juan I. Asensio-Pérez, Yannis Dimitriadis, \\ Sara García-Sastre, Sara Villagrá-Sobrino, Alejandro Ortega-Arranz, \\ Eduardo Gómez-Sánchez, Paraskevi Topali \\ GSIC-EMIC Research group, Universidad de Valladolid, Valladolid, Spain \\ \{kmichos, alex, evi\}@gsic.uva.es, \{juaase, yannis, edugom\}@tel.uva.es, \\ $\{$ saragar, sarena\}@pdg.uva.es
}

\begin{abstract}
The use of Conversational Agents (CAs) in computer-supported collaborative learning (CSCL) has shown promising results regarding students' productive dialogue and learning. Yet, limited work has explored the connection between the configuration of the CA behavior, the nature of the learning task, and the student behavior in authentic educational settings. In this work, we describe a pedagogical design space of CAs for collaborative learning composed of three dimensions: task design, domain model, and agent intervention strategies. We conduct an initial field study in a university classroom comparing two types of agent intervention strategies based on student participation, dialogue, and satisfaction. 54 university students worked in pairs in the same collaborative brainstorming task with a CA tool and were randomly assigned in two CA conditions with a) knowledge-based prompts to connect two domain concepts, b) social prompts to link their partners' contributions. The results show that students who received knowledge-based prompts significantly exchanged more messages with evidence of explicit reasoning and were more satisfied with the agent and their discussion during the task. Students from both conditions reported problems like the lack of context-awareness and timely interventions by the agent. We discuss the relation between the agent intervention strategies and the task design towards seeking design recommendations for CAs in CSCL.
\end{abstract}

Keywords: conversational agents, CSCL, task design, agent intervention strategies, dialogue

\section{Introduction}

Conversational Agents (CAs) have recently gained momentum in different fields such as Healthcare, Marketing, Tourism, and Education and aim to support natural language interaction between humans and computers [20]. This interaction paradigm has a significant potential for applications in Technology Enhanced Learning (TEL) due to the critical role of dialogue as a mediator for active

The final authenticated version is available online at 
learning. For instance, in group settings, CA-based dialogue allows students to elaborate on the learning content, negotiate their opinions and ideas, and argue based on predefined or their own lines of reasoning $[6,1]$. CAs have been explored in TEL under a variety of terms including "pedagogical agents", "learning companions", "chatbots", "virtual assistants", and "dialogue systems" $[20,5]$.

A substantial body of studies conducted in one-to-one learning settings where one student interacts with the agent, suggests that CAs can increase student interaction [18], positively impact student satisfaction and learning outcomes [9], and improve students' comprehension, motivation and engagement [19]. In addition, the use of CAs has emerged in group learning settings where two or more students interact with one or multiple agents with the aim of solving a common learning task. A relatively low number of studies in computer-supported collaborative learning (CSCL) suggests that CA-based activities can enrich students' productive dialogue, impact collaborative learning, and facilitate knowledge exchange through students' explicit reasoning $[17,6]$.

Despite the apparent benefits of CAs in collaborative learning, various problems have been reported concerning the agent efficacy to promote students' collaborative interactions and, thus, their learning. Some of these problems relate to student frustration with the agent, limited student attention to agent prompts, simplified responses to the agent, and lack of fruitful human-to-agent or humanto-human interactions $[12,6]$. The pitfalls of effective CA-based collaborative learning activities have been often associated to inadequate technological or pedagogical design of CAs. Previous work has explored CA design elements concerning the CA behavior. The design aim was to provide appropriate adaptive support for collaborative learning considering the students' contributions to the dialogue, the educational context and students' needs [17, 1, 20, 12]. However, research on the design of CAs for collaborative learning has neglected an important and broader pedagogical design space that could potentially affect the student behavior and agent efficacy. For instance, CA design might involve the adaptation of the CA behavior, according to the pedagogical aims of the learning tasks, or different knowledge domains. Consequently, there is a need for extensive empirical evidence showing the connection between the different pedagogical design choices and the expected student benefits (e.g., students' productive interactions, learning, satisfaction), especially in authentic educational settings.

In this paper, we formulate a space of pedagogical design elements for CAs and focus on one type, namely the agent intervention strategies. In other words, we aim to shed more light to the pedagogical design decisions that affect the CA behavior. We present a field study in a university classroom to understand the influence of the pedagogical design decisions on the effectiveness of CA-based interventions in an authentic educational setting.

\section{Design elements of CAs for collaborative learning}

The design of CAs for collaborative learning has distinct characteristics when compared to those of one-to-one learning settings (i.e., where only one learner in-

The final authenticated version is available online at

https://doi.org/10.1007/978-3-030-57717-9_16 
teracts with a CA) due to students' collaborative work, their social interactions, and their knowledge co-construction process $[3,1]$. The technological design regards elements such as the use of Natural Language Processing techniques, the agent and chat interface design or the use of existing instant messaging tools (e.g., whatsapp, messenger, telegram) [20]. Although the technology used may influence the effective application of CAs in educational practice, in this paper, we focus on the pedagogical design elements of CAs. The challenge lies in the identification of a pedagogical design space that affects the CA behavior and could be addressed by non-technical stakeholders such as teachers or instructional designers. We draw on research in the areas of CAs, CSCL, and Intelligent Tutoring Systems (ITS) and distinguish the following, interrelated pedagogical design elements for CAs (see Fig. 1):

Task design: This design element concerns different pedagogical models for collaborative learning, which involve structuring of social interactions and setting up common goals for the learning partners (i.e., the design of tasks regards the epistemic design [7] and the learning objectives). In the context of CAs, students may be involved in collaborative problem-solving tasks that require the development of both cognitive and social skills, and involve human-to-human or human-to-agent interactions [15].

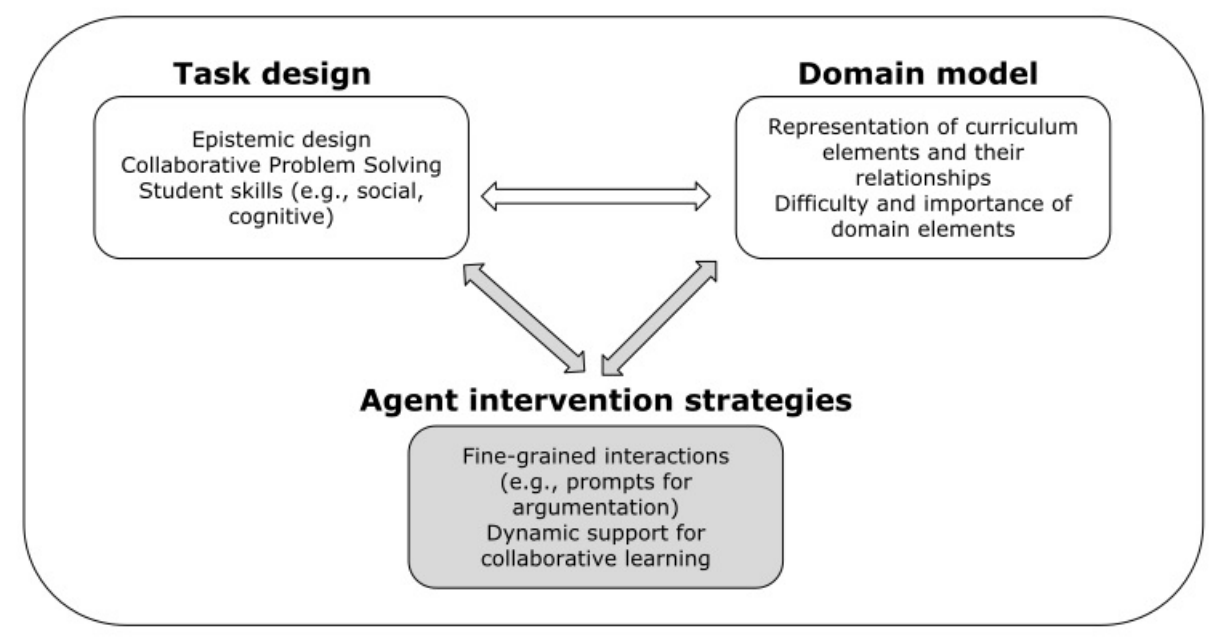

Fig. 1. Design elements of CAs for collaborative learning with focus on the pedagogical agent intervention strategies (grey color).

Domain model: This design element has been extensively used in ITS to represent curriculum knowledge in a given domain. A domain model is a representation of curriculum elements and their relationships (e.g., topics, courses, concepts, procedures). The curriculum elements might have different levels of importance and difficulty for the learners [14].

Agent intervention strategies: This design element defines the fine-grained, student-to-agent or student-to-student interactions (e.g., prompts for ar-

The final authenticated version is available online at 
gumentation or micro-scripts in CSCL [11]). In the context of CAs, the agent intervention strategies take the form of dynamic support because they are interactive and triggered after real time analysis of the student discussion [1]. Some forms of agent interventions include social, cognitive support, student guidance through the task [4] or orchestration [2]. In some cases, CA support follows the guidelines of specific frameworks aiming at promoting student-driven discussions. Following this approach, multiple CSCL research studies explored the use of Academically Productive Talk (APT) moves in collaborative learning [17, 1 , 6] as an effective classroom discourse that can be triggered by CAs. The benefit of APT-based strategies lies on their applicability in different domains and subject materials [6]. The APT-based strategies include knowledge or social-based prompts that facilitate productive talk and the development of students' reasoning [13]. These prompts can be classified along three dimensions: 1) learning community: they encourage students to build on the contributions of their partners with social triggers such as adding information or agreeing and disagreeing: 2) accurate knowledge: they encourage students to make accurate statements or claims that are based on explicit facts, prior knowledge or material taught in the classroom; and 3) rigorous thinking: they encourage students to build rigorous and logical arguments based on evidence.

The eventual success of the CA depends on the effective combination of the different design elements, shown in Fig. 1. In our case, we consider that the domain model and the task design are given, and we explore the effectiveness (assessed in a real context) of two different and relevant agent intervention strategies drawn from research on APT. The empirical evidence may shed some light towards the appropriate design of CAs.

\section{$2.1 \quad$ Research question}

A set of studies has explored the use of the APT framework in CA-based collaborative learning in small groups of students (dyads or triads), but limited work compared two different APT moves in the same task. Some studies compared the move of "Revoice" with that of "Agree-disagree" [1] or the "Revoice" move with the APT "Feedback" support [6]. In both cases, the "Revoice" move was more effective in terms of the intensity of students' interactions and learning (see $[1,6$, 17] for a detailed description of APT-based strategies and APT "Feedback" support). However, the above studies did not compare agent intervention strategies that belong to different APT dimensions and pedagogical objectives (learning community, accurate knowledge, rigorous thinking). In our study, we chose to evaluate two relevant APT-based strategies that have not been analyzed in the previous studies and correspond with two different APT dimensions. The first type of agent intervention strategy is based on accurate knowledge and prompts students to connect two domain concepts ("Build on prior knowledge"). The second type has social characteristics due to the involvement of the learning community and prompts students to link their contributions ("Linking contributions"). These two separate strategies promote the development of either cog-

The final authenticated version is available online at 
nitive (knowledge-based strategy) or social (social-based strategy) skills and can be practiced by students during collaborative learning.

Prior studies reveal different factors which affect the effectiveness of the CA behavior (difficulty of the task, the learning material, the age and developmental stage of the students) and suggest carrying out further research in real educational settings $[1,6,17]$. In addition, those studies adopted different evaluation constructs such as the student dialogue, participation, satisfaction, and learning to understand the agent efficacy. In our case, we carry out a novel comparison between two relevant agent interventions strategies, associated with two APT dimensions, in a brainstorming task and analyze their efficacy through the student dialogue, participation and satisfaction. We use process analysis [1] to understand students' collaborative interactions and focus on the application of the CA-based task in a university classroom. We formulated the following research question:

RQ: To what extent does the design of a collaborative CA task that includes knowledge-based (Build on prior knowledge), as compared to social-based (Linking contributions), agent intervention strategies impact the effectiveness of the task enactment?

In the current paper, we evaluate the effectiveness of the task enactment with the following constructs: a) student participation, b) student dialogue, c) student satisfaction with the task and the agent.

\section{Methodology}

\subsection{Context}

We designed a field study in an authentic educational environment [16] to evaluate the research question. The study took place in a first-year undergraduate university course called "ICT in Education". N=54 students (38 Females, 16 Males), pre-service teachers for Primary Education, participated in the study. Their age ranged between 17-33 years old $(\mathrm{M}=19.15, \mathrm{SD}=2.42)$. We used a $\mathrm{CA}$ technology which allows the configuration and implementation of collaborative tasks in students' pairs. The CA technology has been developed within a European project (www.colmooc.eu) and consists of two parts: a) a configurable agent editor (see Fig. 2-left) which allows teachers to author a collaborative CA task, a domain model and APT-based agent intervention strategies (i.e., the three elements of the design space shown in Fig. 1) - the teacher can thus tailor the agent design to his/her particular knowledge domain and pedagogical intentions; b) a player which allows students to discuss in pairs, interact with the agent and submit a final common answer to the task (see Fig. 2-right).

Based on the CAs' pedagogical design elements described in Section 2 (task design, domain model, agent interventions strategies), one researcher in collaboration with two teachers of the course "ICT in Education", designed a collaborative learning task aligned with a domain topic of the course. In this task, students had to perform a brainstorming activity to discuss and agree on two main benefits and problems of collaborative learning. We leveraged the flexibility

The final authenticated version is available online at

https://doi.org/10.1007/978-3-030-57717-9_16 


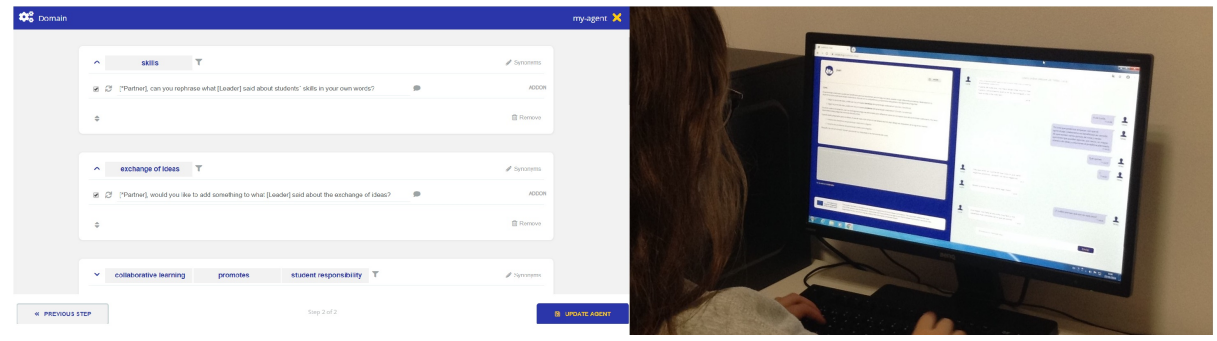

Fig. 2. Conversational agent tool: editor (left) and player (right)

provided by the $\mathrm{CA}$ tool in order to design one agent with a clear dominance of knowledge-based interventions and another one that consists of social-based interventions.

\subsection{Research design}

To evaluate and compare the two different APT-based agent intervention strategies, we conducted a between-subjects (also termed between-groups) research design. Accordingly, students were randomly assigned to one of the following CA conditions (see Fig. 3): Knowledge Support Agent (KSA) and Social Support Agent (SSA). In order to control the other two design elements (task design, domain model), students in both conditions had to perform the same brainstorming task in pairs and the agent interventions were triggered with the same domain words. In the former condition (KSA), the agent acted with knowledgebased prompts and asked students to explain how two domain concepts relate to each other (see Table 1). In the latter condition (SSA), the agent acted with social-based prompts and asked students to add information on their partners' contributions. In both conditions, the agent interventions were directed to one student and were triggered when a domain word was written by his/her partner. Six agent interventions were configured. The CA tool followed some rules to trigger interventions based on the real-time analysis of the students' messages (e.g., the same interventions were not repeated and a 90" time limit was set between two interventions).

Table 1. APT-based agent intervention strategies used in the study

\begin{tabular}{|l|l|}
\hline Agent intervention strategy & Example \\
\hline Knowledge Support Agent (KSA) & $\begin{array}{l}\text { Alex, do you think that the development } \\
\text { of students' skills is somehow related to } \\
\text { collaborative learning? }\end{array}$ \\
\hline $\begin{array}{l}\text { Social Support Agent (SSA) } \\
\text { APT move: Linking contributions-AddOn }\end{array}$ & $\begin{array}{l}\text { Alex, would you like to add something to } \\
\text { what Maria said about the development } \\
\text { of students' skills? }\end{array}$ \\
\hline
\end{tabular}

Before carrying out the collaborative task, two instructors demonstrated the $\mathrm{CA}$ tool and instructed students to perform the activity in 30 minutes. The study took place in two consecutive sessions. In both sessions, $\mathrm{N}=28$ students

The final authenticated version is available online at 
were randomly assigned to KSA condition and $\mathrm{N}=26$ students were assigned to the SSA condition.

\subsection{Research instruments}

Before the collaborative task, students responded to a pre-questionnaire including 2 demographic questions (age, gender), one question about their familiarity with chat technologies and one question about their previous use of CAs.

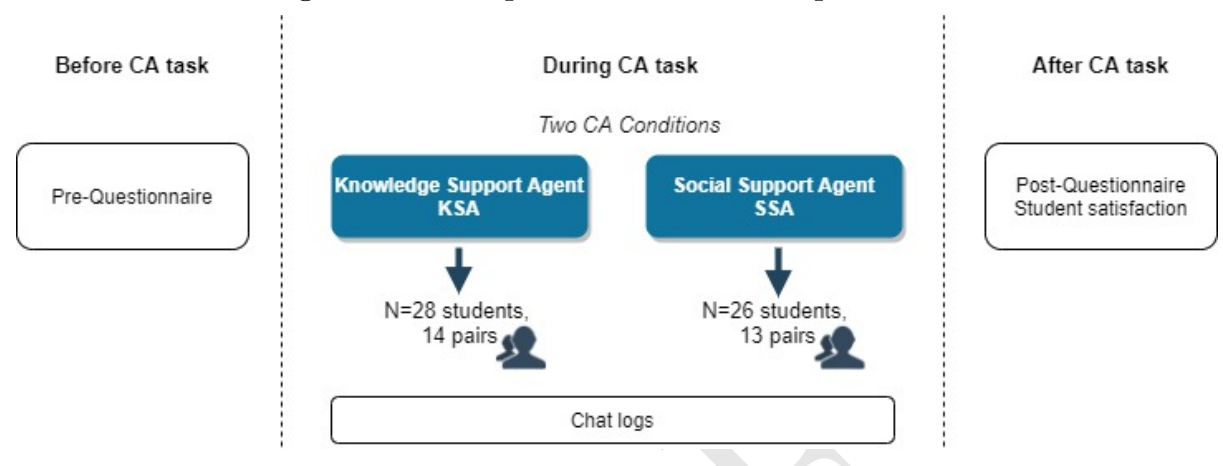

Fig. 3. Research study design in two CA conditions

To evaluate students' participation and their dialogue, we used the chat logs of the CA tool. We performed a content analysis of the messages to further understand student participation and social interactions related to learning. We used the coding scheme defined by [17], since it is relevant for collaborative problem solving activities and has been already applied in the context of CAs [17]. Table 2 presents the coding scheme used in the study.

Four researchers (authors of the paper) divided the corpus of messages $(\mathrm{N}=1604)$ in four parts and performed the content analysis using the message as unit of analysis. One researcher reviewed all the messages of the other coders and after discussion coders reached full interrater agreement (100\%).

To evaluate student satisfaction with the task and the CA, we used a postquestionnaire. The post-questionnaire included 3 questions (5-Likert scale) about student satisfaction with the task (see Fig. 6) and 4 questions (5-Likert scale) about student satisfaction with the CA (see Fig. 7). In addition, three open questions were used to elicit students' comments regarding positive, negative experiences and recommendations about the CA task. Mann-Whitney's U tests for independent samples were calculated to understand significant differences between median values in each CA condition. This test was selected because we used continuous or ordinal variables in each evaluation construct (number of messages, time, Likert scale responses), which were not normally distributed.

\section{Results}

Based on the pre-questionnaire data, students in both conditions were familiar with chat technologies whereas students had rarely used CAs (almost never or

The final authenticated version is available online at

https://doi .org/10.1007/978-3-030-57717-9_16 
Table 2. Coding scheme used in the study based on [17]

\begin{tabular}{|l|l|}
\hline Category & Description \\
\hline Off task & $\begin{array}{l}\text { Contributions that do not relate to the task and } \\
\text { often play a purely social function (e.g., } \\
\text { "Hi Juan") }\end{array}$ \\
\hline Repetition & $\begin{array}{l}\text { Reiterations of prior contributions often } \\
\text { repeated after some time for a better understanding. }\end{array}$ \\
\hline Team management & $\begin{array}{l}\text { Management-oriented utterances used for task } \\
\text { coordination }\end{array}$ \\
\hline Common understanding & $\begin{array}{l}\text { Short utterances used to establish common } \\
\text { understanding on the subject (e.g., "Ok") }\end{array}$ \\
\hline Issue & $\begin{array}{l}\text { What needs to be done or resolved to proceed with } \\
\text { the overall task }\end{array}$ \\
\hline Position & $\begin{array}{l}\text { Opinions usually related to the resolution of the } \\
\text { issue raised }\end{array}$ \\
\hline Argument & $\begin{array}{l}\text { Opinions supporting or objecting to a position } \\
\text { (e.g., "You are absolutely right") }\end{array}$ \\
\hline Explicit position & $\begin{array}{l}\text { Positions that explicitly outline reasoning } \\
\text { on domain concepts }\end{array}$ \\
\hline Explicit argument & $\begin{array}{l}\text { Much as explicit positions, arguments displaying } \\
\text { explicit reasoning on domain concepts }\end{array}$ \\
\hline
\end{tabular}

sometimes), and there were no significant differences between the two conditions. Thus, we assume that students' familiarity with chat technologies and previous use of CAs did not influence our results.

\subsection{Student and agent participation}

The student and agent participation during the collaborative activity was analyzed through the chat logs. First, the time spent to complete the task (see Fig. 4) was measured in order to compare the number of agent interventions and the number of student messages in each condition. Results show that although students in KSA condition employed on average more time (28.76 min) than participants in SSA condition (25.44 min), there were no significant differences between the two conditions $(\mathrm{p}=.1852)$. The activity was designed to last for 30 minutes, being the median value of KSA condition closer to the estimated value (28.1 $\mathrm{min}$ ) as compared with SSA (21.8 $\mathrm{min})$. The difference in the time to complete the task is also explained by the higher number of messages exchanged by students in KSA condition and the higher number of agent interventions (see Fig. 5).

During the collaborative task, 65 agent interventions were triggered in all student pairs and addressed one partner of a pair each time. In KSA condition, 37 agent interventions were triggered and ranged between 1-4 interventions $(\mathrm{M}=2.64, \mathrm{SD}=1.008)$ per student pair. In SSA condition, 28 agent interventions were triggered and ranged between 1-3 interventions $(\mathrm{M}=2.15, \mathrm{SD}=.555)$ per student pair. Students in KSA condition significantly $(\mathrm{p}=.0070)$ submitted more messages to their partners (median value $=30$ messages) than participants 


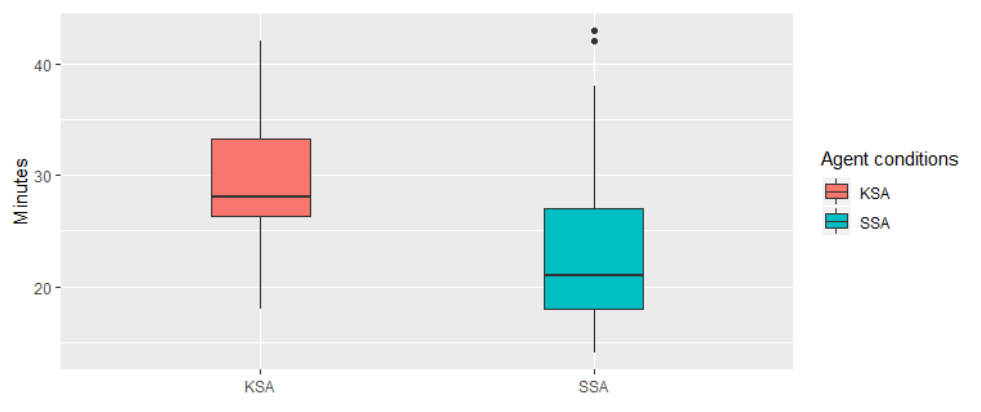

Fig. 4. Task duration in each CA condition

in SSA condition (median value $=21$ messages). An analysis of the number of words written as answers to the agent questions shows that students' answers in KSA were significanlty longer (median value $=26$ words) than the answers written in SSA (median value $=17.5$ words, $p=.0012$ ). Students who were not asked by the CA were not included in this analysis ( 3 and 7 participants from KSA and SSA respectively).
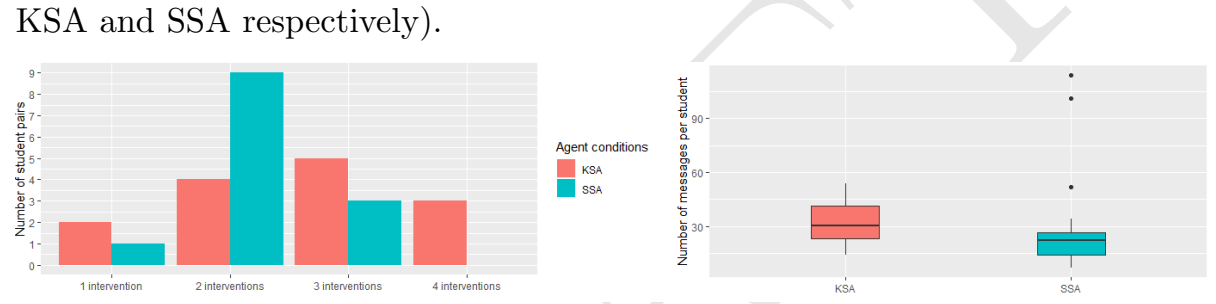

Fig. 5. Number of agent interventions per student pair in each CA condition (left), and number of messages per student in each CA condition (right)

\subsection{Student dialogue}

A content analysis of student dialogue was used to better understand student participation and social interactions related to learning. Table 3 presents the content analysis results. Considering the coding scheme [17], in both conditions the higher percentage of messages was related to team management (meaning that students were coordinating themselves for the task), and common understanding (e.g., short messages like "Ok"). In KSA condition, students' team management messages were more frequent than in SSA. One interpretation is that knowledgebased interventions increased students' effort to coordinate themselves for the task whereas social-based interventions required less team management. Some coded categories appeared in exactly the same or similar percentage of messages in both conditions. These categories were issue (messages referring to issues in order to resolve the task), position (opinions related to resolution of the issue raised), and argument (opinions supporting or objecting a position).

However, students wrote more frequently messages irrelevant to the task (off task) in SSA condition as compared the KSA condition. In SSA condition, students repeated more often previous contributions in the discussion after some

The final authenticated version is available online at 
Table 3. Content analysis results in the two CA conditions (KSA-Knowledge Support Agent, SSA-Social Support Agent)

\begin{tabular}{|l|l|c|l|c|l|c|}
\hline Category & \multicolumn{3}{l}{ KSA } & \multicolumn{2}{l|}{ SSA } & \multicolumn{2}{l|}{ Both groups } \\
\hline & $N$ & Percentage (\%) & $N$ & Percentage (\%) & $N$ & Percentage (\%) \\
\hline Explicit argument & 43 & 5 & 8 & 1 & 51 & 3 \\
\hline Explicit position & 66 & 7 & 24 & 3 & 90 & 6 \\
\hline Issue & 87 & 10 & 71 & 10 & 158 & 10 \\
\hline Argument & 92 & 10 & 75 & 11 & 167 & 10 \\
\hline Repetition & 44 & 5 & 78 & 11 & 122 & 8 \\
\hline Position & 116 & 13 & 86 & 12 & 202 & 13 \\
\hline Off task & 100 & 11 & 106 & 15 & 206 & 13 \\
\hline Common understanding & 140 & 16 & 120 & 17 & 260 & 16 \\
\hline Team management & 211 & 23 & 137 & 19 & 348 & 22 \\
\hline
\end{tabular}

time (repetition) compared to KSA. These unrelated students' messages and their repetitions in SSA could be explained by the results on the two final categories. In KSA condition, students showed more evidence of explicit reasoning than in SSA, meaning that students demonstrated higher levels of reasoning on the domain concepts. In KSA, students also showed more evidence of explicit arguments compared to SSA, meaning that students supported or opposed the contributions of their partners with reasoning. These final two categories (explicit position, explicit argument) have been found to show stronger relations to student learning during collaborative activities $[17,6,1]$. Thus, our results suggest that students who received knowledge based-prompts during the brainstorming task participated more and their dialogic interactions were more relevant to learning. The following results regarding students' perceptions and their satisfaction provided more clues regarding their participation and dialogue.

\subsection{Student satisfaction with the task and the agent}

We analyzed student satisfaction with the task and the agent using both quantitative and qualitative self-reported data from the post-task questionnaire. According to the results, students in the KSA condition were more satisfied with their discussion during the collaborative activity compared to SSA condition (see Fig. 6). This result aligns with the positive comments provided by students in KSA condition because 13 out of 28 students pointed out that the collaborative CA task helped them to develop more arguments, exchange opinions/ideas and reasoning on the topic (e.g., "The agent helped to develop or think new arguments about collaborative learning through its questions"). This is also consistent with the content analysis results due to the higher levels of explicit reasoning and argumentation in the KSA condition, as explained above. Students highly agreed in both groups that the collaborative activity was beneficial for their learning and they would like to participate in more activities with CAs in the future.

The final authenticated version is available online at

https://doi.org/10.1007/978-3-030-57717-9_16 

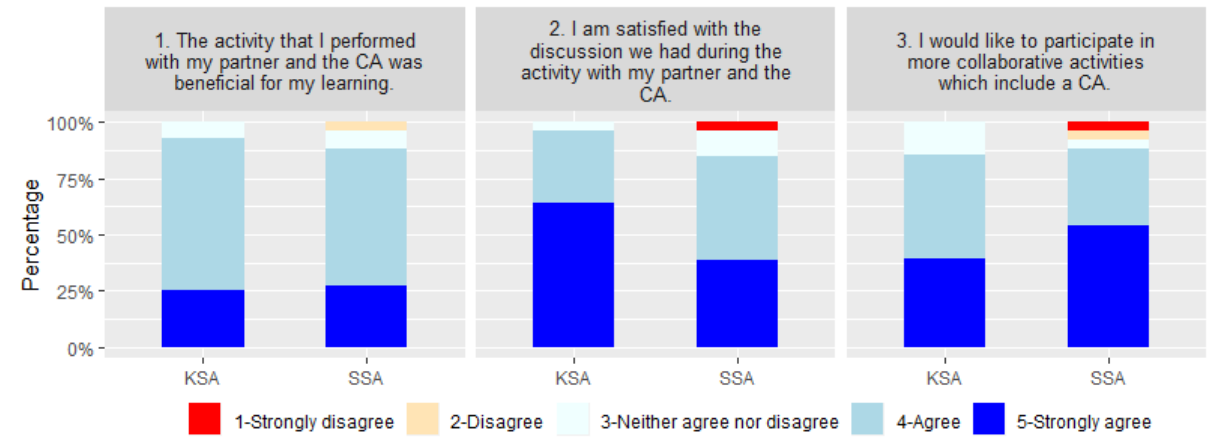

Fig. 6. $100 \%$ stacked bars regarding student satisfaction with task from KSA $(\mathrm{N}=28)$ and SSA $(\mathrm{N}=26)$ condition based on self-reported data on the post-task questionnaire

Students' answers provided in the final questionnaire are in line with the previous quantitative results. Students were more satisfied in KSA condition with the help provided by the agent regarding reasoning on key aspects of collaborative learning, exchanging arguments and solving the task (see Fig. 7). This is also confirmed by their responses in the open questions (e.g., "It helps you to solve the task more easily since answering the agent's questions made you think easily about the answer of the task"). However, more students in KSA condition pointed out that the agent interrupted their discussion (e.g., "The conversational agent should not ask the questions in the middle of the discussion with the other partner"). The higher number of agent interventions in this group, and the fact that students were trying to coordinate more among themselves for the task (team management) could explain the stronger perception of agent interruption.

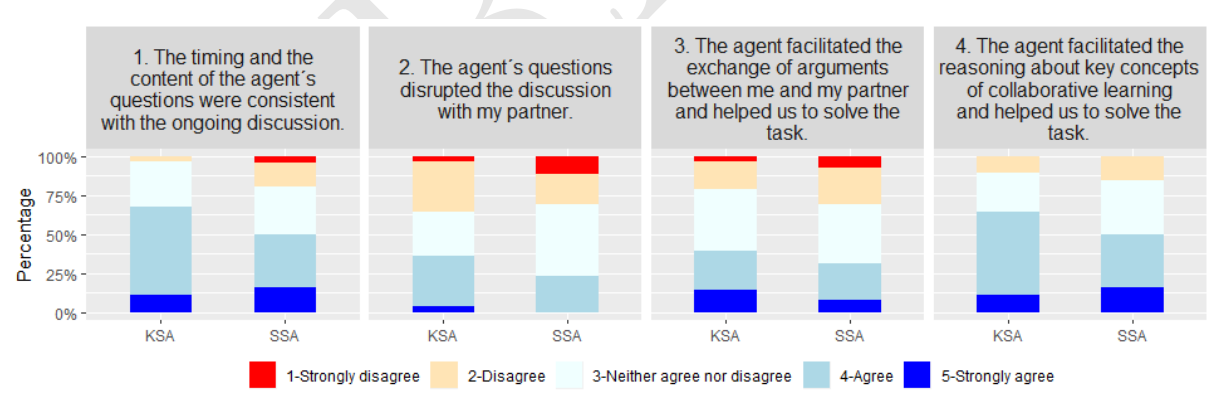

Fig. 7. 100\% stacked bars regarding student satisfaction with agent from KSA (N=28) and SSA $(\mathrm{N}=26)$ condition based on self-reported data on the post-task questionnaire

Students provided recommendations for future activities with CAs. Students in both conditions mentioned that the agent should ask more questions showing their interest to interact more with the agent. Students also pointed out that the agent should ask both partners because in some cases the agent interacted with only one student. Last, students mentioned the need of timely and context-aware interventions by the agent (e.g., "The conversational agent should appear right on issues we are dealing with").

The final authenticated version is available online at

https://doi.org/10.1007/978-3-030-57717-9_16 


\section{Conclusions and Future Work}

In this paper, we formulated a space of interrelated pedagogical design elements (task design, domain model, agent intervention strategies) with the aim to contribute to research related to CAs for CSCL. Among the design elements, we focused our empirical research on the agent intervention strategies and built on previous CSCL studies that examine the agent behavior based on the APT framework and the expected benefits to students (social interactions, learning, satisfaction).

We investigated the extend to which the design of a collaborative CA task impacts the effectiveness of the task enactment when two different types of agent intervention strategies (knowledge and social-based) are employed. The two agent intervention strategies are based on two different APT objectives ( $a c$ curate knowledge, learning community) and their comparison has not been explored in previous studies $[17,1,6]$. We found that the knowledge support agent condition provided better indicators regarding student participation, content of the student dialogue and student satisfaction. First, student participation was significantly higher in terms of messages exchanged and interactions as replies to the agent. This result reveals that a knowledge-support agent was either a better strategy to increase the intensity of student activity and interactions during the collaborative task or demanded higher student effort towards accomplishing the task (or both). Second, the content analysis of the student dialogue on the one hand shows that student interactions were more relevant to learning due to the higher levels of explicit reasoning and arguments [17] but on the other hand it required more team management. The predominance of team management student behavior may be connected with higher collaborative cognitive load in group settings as explained in [10]. Finally, students were more satisfied with their discussions with the knowledge support agent but reported more frequently disturbing interruptions by the agent. In both conditions, the students mentioned a certain level of disruption and lack of context-awareness and timely agent interventions.

Our results suggest that the effective combination of the different design elements, formulated and shown in Fig. 1, may determine the meaningful CA behavior for collaborative learning interactions. During the process of defining the task design, the learning objective (e.g., students are required to reach consensus or students are asked to create a common solution) and the student context (e.g., students who know each other or students with prior knowledge on the domain) would influence the design choices for different types of agent interventions strategies. In our study, students had to perform a brainstorming task and a knowledge support agent (as a third partner) added more concepts on the discussion which may be aligned with the brainstorming task objectives, although, students had to dedicate higher effort to collaborate. Based on the aforementioned conclusions, the effective CA design for collaborative learning may require a combination of different pedagogical agent behaviors [8] that best adapt to the nature of the task and the student behavior (e.g., students' contributions to the dialogue).

The final authenticated version is available online at

https://doi.org/10.1007/978-3-030-57717-9_16 
The reported study has several limitations. Students knew each other and this could have somewhat reduced students' appreciation for the social support agent. Another study might investigate a social support agent with pairs who do not know each other (e.g., in a MOOC context). In addition, students may perceive more competent (or intelligent) an agent that employs knowledge-based strategies as compared to social-based strategies. The use of APT-based agent interventions highly depends on the domain model. Future studies may consider advanced learning analytics techniques (e.g., modeling cognitive states) to detect student behavior and improve the agent context-awareness. However, as far as it concerns non-technical stakeholders (e.g., teachers), there is a trade-off between the advanced "agent powers" (student behavior modelling and agent interventions) and a possible "design burden" for teachers to understand and configure advanced agent behaviors during the design process. These suggestions further show the importance of the pedagogical design elements and the student context and align with previous research on the different factors for the design of CAs for collaborative learning (difficulty of the task, learning material, age and developmental stage of students) $[1,6,17]$.

The pedagogical design space described in this paper requires further research studies to understand the relations between the CA design elements and their connection with student learning. As future work, we identified relevant areas that will eventually shed more light on the appropriate CA design. We are studying different task types of Collaborative Problem Solving (CPS) [15] as a basis for comparing task designs and conduct co-design sessions with teachers who implement CA tasks in their university courses. Finally, we are planning to conduct research in remote learning contexts and in particular with synchronous collaborative CA activities in MOOCs and online courses.

\section{Acknowledgements}

Authors would like to thank Alejandra Martínez-Monés who assisted in the study and Stavros Demetriadis, Tasos Karakostas, and Stathis Nikolaidis who provided the CA technology, in the context of project grant 588438-EPP-1-20171-EL-EPPKA2-KA funded by the European Commission, which supported this research. Further support was provided by project grant TIN2017-85179-C3-2-R funded by the Spanish State Research Agency (AEI) and the European Regional Development Fund and project grant VA257P18 funded by the Regional Government of Castilla y León and the European Regional Development Fund.

\section{References}

1. Adamson, D., Dyke, G., Jang, H., Rosé, C.P.: Towards an agile approach to adapting dynamic collaboration support to student needs. International Journal of Artificial Intelligence in Education 24(1), 92-124 (2014)

2. Amarasinghe, I., Hernández Leo, D., Manathunga, K., Jonsson, A.: Sustaining continuous collaborative learning flows in MOOCs: orchestration agent approach. Journal of Universal Computer Science. 2018; 24 (8): 1034-51. (2018)

The final authenticated version is available online at

https://doi.org/10.1007/978-3-030-57717-9_16 
3. Bittner, E., Oeste-Reiß, S., Leimeister, J.M.: Where is the bot in our team? Toward a taxonomy of design option combinations for conversational agents in collaborative work. In: Proceedings of the 52nd Hawaii International Conference on System Sciences. pp. 284-293 (2019)

4. Bittner, E.A., Küstermann, G.C., Tratzky, C.: The facilitator is a bot: Towards a converstational agent for facilitating idea elaboration on idea platforms. In: Proceedings of the 27th European Conference on Information Systems (ECIS) (2019)

5. Colace, F., De Santo, M., Lombardi, M., Pascale, F., Pietrosanto, A., Lemma, S.: Chatbot for e-learning: A case of study. International Journal of Mechanical Engineering and Robotics Research 7(5), 528-533 (2018)

6. Dyke, G., Adamson, D., Howley, I., Rosé, C.P.: Enhancing scientific reasoning and discussion with conversational agents. IEEE Transactions on Learning Technologies 6(3), 240-247 (2013)

7. Goodyear, P., Carvalho, L.: The architecture of productive learning networks. Abingdon: Routledge (2014)

8. Hayashi, Y.: Multiple pedagogical conversational agents to support learner-learner collaborative learning: Effects of splitting suggestion types. Cognitive Systems Research 54, 246-257 (2019)

9. Huang, J.X., Lee, K.S., Kwon, O.W., Kim, Y.K.: A chatbot for a dialogue-based second language learning system. In CALL in a climate of change: Adapting to turbulent global conditions, short papers from EUROCALL 2017 pp. 151-157 (2017)

10. Kirschner, P.A., Sweller, J., Kirschner, F., Zambrano, J.: From cognitive load theory to collaborative cognitive load theory. International Journal of ComputerSupported Collaborative Learning 13(2), 213-233 (2018)

11. Kobbe, L., Weinberger, A., Dillenbourg, P., Harrer, A., Hämäläinen, R., Häkkinen, P., Fischer, F.: Specifying computer-supported collaboration scripts. International Journal of Computer-Supported Collaborative Learning 2(2-3), 211-224 (2007)

12. Kumar, R., Rosé, C.P., Wang, Y.C., Joshi, M., Robinson, A.: Tutorial dialogue as adaptive collaborative learning support. Frontiers in Artificial Intelligence and Applications 158, 383 (2007)

13. Michaels, S., O'Connor, C., Resnick, L.B.: Deliberative discourse idealized and realized: Accountable talk in the classroom and in civic life. Studies in Philosophy and Education 27(4), 283-297 (2008)

14. Murray, T.: An overview of intelligent tutoring system authoring tools: Updated analysis of the state of the art. In: Authoring tools for advanced technology learning environments, pp. 491-544. Springer (2003)

15. OECD: Draft collaborative problem solving framework (2015), Retrievedfromhttp://www.oecd.org/pisa/pisaproducts/ DraftPISA2015CollaborativeProblemSolvingFramework.pdf

16. Saldaña, J.: Thinking qualitatively: Methods of mind. Sage Publications (2014)

17. Tegos, S., Demetriadis, S., Karakostas, A.: Promoting academically productive talk with conversational agent interventions in collaborative learning settings. Computers \& Education 87, 309-325 (2015)

18. VanLehn, K., Graesser, A., Jackson, G., Jordan, P., Olney, A., Rosé, C.: Natural language tutoring: A comparison of human tutors, computer tutors, and text. Cognitive Science 31(1), 3-13 (2007)

19. Veletsianos, G., Russell, G.S.: Pedagogical agents. In: Handbook of research on educational communications and technology, pp. 759-769. Springer (2014)

20. Winkler, R., Söllner, M.: Unleashing the potential of chatbots in education: A stateof-the-art analysis. In Academy of Management Annual Meeting (AOM) (2018)

The final authenticated version is available online at

https://doi.org/10.1007/978-3-030-57717-9_16 